\title{
A CRT graphics system for experimental research
}

\author{
DANIEL B. KAYE and EDWARD H. HERSKOVITS \\ University of Califormia, Los Angeles, California
}

\begin{abstract}
A graphics system to create, display, store, and print stimuli in a hardware-enhanced, microcomputer-driven CRT environment is described. This graphics system is compared with other computer-based systems, as well as with systems requiring the use of tachistoscopes.
\end{abstract}

Modern computer graphics systems have the potential to replace the tachistoscope as the primary tool in research on visual information processing. Among the limitations of the tachistoscope, Glazenborg and Schreuder (1983) noted that it (1) is inaccurate in timing because of the mechanical shutters used in projector systems, (2) requires simple stimulus sequences because of the relatively small number of visual channels (usually three-six), and (3) cannot be used easily to present pictures that are perceived entirely in the foveal visual field because of constraints on visual angle. Unfortunately, problems must be solved before the transition to the use of computer graphics can be completed.

\section{DESIRABLE CHARACTERISTICS}

The ideal computer graphics system for experimental research should have certain minimal hardware and software features. At input, there should be routines to facilitate the encoding of stimuli into the computer. Output should include hard copy (e.g., printer, plotter) to reproduce screen attributes by means of shading, color, stippling, etc. The visual display needs sufficient resolution to handle relatively complex stimuli (e.g., pictures, alphanumeric characters). Multiple colors, shades of gray, and programmable intensity for each pixel also are valuable features.

The system should contain enough resident memory to be able to present a sequence of stimuli in rapid succession; the display memory should be accessible so that it may be programmed to perform customized routines. The system should be able to generate or refresh images in several milliseconds or less. Real-time image transformation (e.g., rotation, rescaling) should be possible, and image decay time should be brief.

This research was funded in part by Biomedical Research Support Grant 4-523428-24739 awarded to the first author. Portions of this paper were presented at the annual meetings of the American Psychological Association, Anaheim, CA, 1983. Comments on earlier drafts by Lisa Kahan, Elizabeth Bjork, Duane Buhrmester, and Tim Post are greatly appreciated. Send reprint requests or inquiries about software to: Danjel B. Kaye, Department of Psychology, University of California at Los Angeles, Los Angeles, CA 90024.
The disk capacity should be large enough that a library of visual stimuli may be maintained on-line for call-up during program operation, and stimulus data files should be transportable between graphics systems. For both input and output routines, the software should be powerful but also user-friendly. Menu-driven routines and instruction code that can be embedded in programs to control experiments are essential.

\section{HIGH-COST SYSTEMS}

Two high-cost graphics systems incorporate some of these characteristics. Glazenborg and Schreuder's (1983) system depends on a Vector General CRT (cost exceeding $\$ 60,000$ ), a vector display system that allows for rapid generation, refresh, and transformation of images. Although Glazenborg and Schreuder highlighted the relative advantages of their software package, the hardware requirements limit the system's accessibility to a few laboratories. Montgomery and Soo Hoo (1982) described a similar system that uses a stand-alone Tektronix 4081 system in which stimuli are vector plotted with rapid refresh; thirty-five 130-vector images can be presented at $40 / \mathrm{sec}$. This speed is fast enough for animation, but it is not fast enough for rapid sequencing of stimuli required in some perception experiments in which images may need to follow one another within several milliseconds. Their input/output routines, written in FORTRAN, are for the most part machine-dependent. This system also is relatively expensive.

\section{ALTERNATIVE LOWER COST SYSTEMS}

A sampling of available graphics boards demonstrates that hardware features tend to vary proportionately with cost in other graphics systems. The CSD "Ultra-Res" graphics board available for S-100 and IBM systems is capable of displaying $1,024 \times 1,024$ pixels on a single page with a drawing time of $1.25 \times 10^{6}$ pixels $/ \mathrm{sec}$. Lower resolution with multiple pages is an option. The Cromemco Dazzler graphics system has high-resolution black/white $(\mathrm{b} / \mathrm{w})$ or color graphics $(650 \times 480)$ with many pages of memory and, given the requirements out- 
lined is one of the better systems, but the cost of the computer, graphics hardware, and monitor may well exceed $\$ 10,000$. The Vectrix V128 system is a high-resolution $(672 \times 480)$ color system with many desirable software and hardware features, but with monitor and without the computer the price exceeds $\$ 3,000$. The CAT -100 graphics boards from Digital Graphics also offer a wide range of graphics features at moderate to high cost.

There are a host of graphics boards available for the IBM PC and similarly priced microcomputers ranging from low-resolution $320 \times 200$ (for color graphics-e.g., the Multidisplay Card by Paradise Systems) to mediumresolution $(640 \times 200 \mathrm{~b} / \mathrm{w}$ version of the same board, or a variety of four-color boards) to high-resolution boards (e.g., 1,024 × 1,024 Artist I by Control Systems; 640 $\times 480$ PC640 by Scion; and $512 \times 480$ Vector Scan 512). Prices vary accordingly, from $\$ 595$ for the Paradise Systems board to $\$ 2,495$ for the Artist I. Because cost of the monitor is a function of the resolution required, some of these systems (e.g., Artist I) would require a substantial total investment. As listed in the manual for the IBM PC, the monochrome resolution is $720 \times 350$, but the refresh cycle of $50 \mathrm{~Hz}$ (every $20 \mathrm{msec}$ ) and the slowly decaying green (P39) phosphor create additional problems for a tachistoscope-emulation system. Software packages are available and typically are focused on computer-aided design (CAD) systems, which are becoming increasingly available for microcomputers but do not boast fast drawing or presentation times, and thus the software would need to be configured for the kinds of applications described in this paper. Cost of software is additional in most cases.

The Apple IIc, a lower cost system, has "double hiresolution" graphics $(512 \times 192$ resolution) with $128 \mathrm{~K}$ built-in memory for rapid swapping of images to the screen. Again, software is additional. One of the more impressive graphics computers to come out recently is the Acorn microcomputer (BBC), which has a price under $\$ 1,000$ without monitor. Included is a medium-resolution four-color graphics $(640 \times 200)$ board (limit of $32 \mathrm{~K})$ with a single page of memory. By mapping colors into the background, multiple images may be stored in the same area and presented as two to three pages of graphics at reduced resolution $(360 \times 200 \mathrm{each})$. Software is limited, with built-in functions for drawing lines, filling triangles, and choosing and changing colors.

This brief review represents the types of systems available to produce reasonable graphics at low to moderate cost. Consideration of a low-, medium-, or high-priced system needs to include the graphics attributes discussed in this paper, as well as the laboratory functions that each microcomputer meets.

\section{THE SELANAR SYSTEM}

We use another system, which is not ideal but has many of the desired characteristics mentioned earlier and is available for modest cost (under $\$ 5,000$, not including the hard-copy devices). A Selanar SG900 graphics board
(Selanar Corporation) is housed in a TeleVideo 950 intelligent terminal. This graphics subsystem is driven by a Z80A-based $64 \mathrm{~K}$ microcomputer with dual doubledensity floppy disks and is interfaced with the computer via a digitizer pad for image generation and a printer and $x-y$ plotter for image hard copy. ${ }^{1}$

The Selanar graphics board provides the system with two pages of on-board memory and a resolution of 700 (horizontal) $\times 250$ (vertical) pixels, allowing displays of complex pictures. Two line drawings, a lion and a violin, are shown in Figure 1. Since the Selanar board is driven by the TeleVideo's noninterlaced raster scan monitor, each display is refreshed every $1 / 60$ th $\sec (60 \mathrm{~Hz})$. Image generation time is determined by (1) the speed of transmission from the host computer to the Selanar board via the RS-232 serial interface, (2) the time for access of data from the disk storage system, and (3) the time for translation into the coordinate system. For example, the lion in Figure 1 was generated from disk in approximately $900 \mathrm{msec}$ (0.3-sec access time, 0.6-sec drawing time). The image may be displayed as it is generated or stored on the appropriate page with the video screen off until display is desired. In the latter case, image generation takes 3-4 msec (although the refresh cycle still is $16.67 \mathrm{msec}$ ). The ability to turn the video screen off allows presentation of complex stimuli without painting them on the screen. The P4 phosphor of the TeleVideo $b / w$ monitor provides rapid image decay.

Stimuli are stored as sets of vectors; the amount of disk storage required by each data set depends on the options used to generate the image. The lion in Figure 1 takes

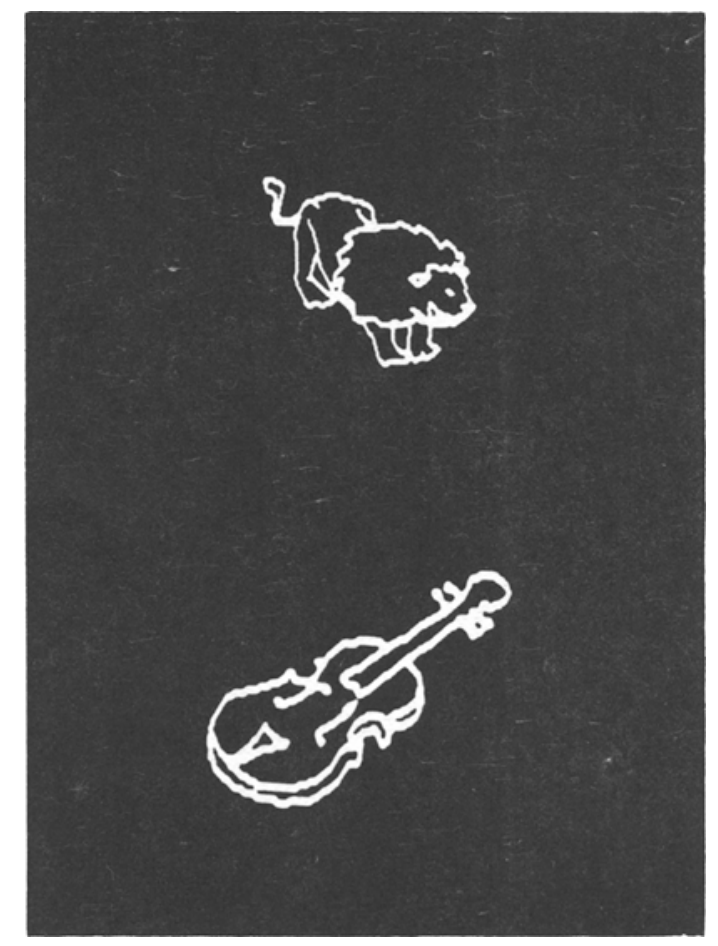

Figure 1. Photographs of two line drawings as they appear on the CRT. 
$1 \mathrm{~K}$ of disk space. Thus, even floppy disk systems can be used to store large libraries of stimuli.

The Selanar system has several environments enabling the user to emulate a Tektronix 4014 terminal, set display attributes (such as video on/off, scaling, zoom), and write alphanumerics to either of the two graphics pages provided. These environments are software-accessible and are used extensively in programs that generate and display images. A library of routines (not included with the hardware) drives the Selanar system and the accompanying peripherals (graphics tablet, plotter, printer). The software package is written in a $\mathrm{CP} / \mathrm{M}$-compatible version of Pascal, but it is hardware-dependent in the sense that it has been written for the Selanar SG900 board and a specific graphics tablet and plotter. Reconfiguration to different tablets or plotters requires minor modifications to the software. Thus, the software is readily transportable to similarly configured microcomputer systems. At present, the software package is used to run perception experiments; the package can be made general so that any feasible tachistoscope-emulation experiment may be run in a user-friendly command environment.

\section{Selanar System Details}

Before generating any image, various graphics parameters are SET by software as needed: (1) The ORIGIN may be relocated; (2) $x-y$ GAIN can be altered to change character size and shape, which can overcome the inherent asymmetry in horizontal $\times$ vertical resolution; (3) basic CHARACTER SIZE (at lowest gain value) can be varied from $0.195 \times 0.160$ in. (vertical $\times$ horizontal) to $0.11 \times 0.09$ in. (when GAIN and CHARACTER SIZE are varied together, characters of many shapes and sizes can be created); (4) the VIDEO screen may turned ON or OFF; (5) any of 12 VECTOR TYPEs may be selected; and (6) the stimulus may be presented automatically for a fixed duration ranging from 16 to $4,000 \mathrm{msec}$ (in $1 / 60$ th-sec increments) by altering DISPLAY TIME. At any time during the data-entry process, these attributes can be changed or the image can be CLEARed.

The primary data-entry mode employs the procedure TRACE. ORIGIN and x-y GAIN are set first. Hard copy then is translated via a digitizer pad (graphics tablet) to a series of $x-y$ coordinates, and the image is displayed simultaneously on the screen.

An alternative mode for data entry is POINT PLOT. The user draws pictures on the screen via the keyboard number pad, thus obviating a digitizer pad. The image is modified by the PEN UP and PEN DOWN commands and WRITE and ERASE (for editing) modes. The pen may be moved in any of eight directions. (Data may be entered with a joystick with the cross-hair option in Selanar, although this option has not been implemented in the software package.)

GEOMETRY is a set of subroutines used to generate displays of CIRCLEs, ARCs, regular POLYGONs, RECTANGLEs, and LINEs.
GEOMETRY, POINT PLOT, and TRACE all allow the user to draw lines in any of three modes: OR (all of the character's pixels are "on"), XOR (all of the character's pixels are "on" except for those that were already "on" before writing the character), and NOT (all of the character's pixels are "off," i.e., erase mode). All three procedures have a mode to FILL any enclosed area with white, solid shading.

ALPHA mode allows the user to generate alphanumeric characters of variable size on the graphics screen. This procedure is necessary because characters must be generated in graphics mode to become part of the final graphics image.

Images produced by any of the above means can be STOREd on disk for future CALLs from a program or for PRINTouts or PLOTs for hard-copy versions.

\section{Evaluation}

The Selanar system scores favorably in terms of the desired characteristics listed earlier. Input is facilitated by the graphics tablet, keyboard, and joystick. Hard copy on both printers and plotters gives excellent reproduction of screen images. The resolution of the Selanar board is sufficient for most applications, and it matches the resolution of most high-resolution, relatively low-cost graphics sytems. Glazenborg and Schreuder (1983) noted that it is sometimes difficult to present pictures foveally on a tachistoscope. The scaling feature of the Selanar system is used to present pictures subtending almost any visual angle with reasonable viewing distances; however, the resolution of the screen provides a lower limit on the size of a stimulus that is recognizable. The Selanar board allows only for black-and-white images, with an option for "filling" (in white) any enclosed area. Intensity is varied manually on the TeleVideo terminal. There are no shades of gray or multiple colors. Similar systems, such as Scion's Microangelo and Digital Graphic Systems' CATseries allow for these, but at greater cost.

Two pages of resident memory constrain the type of stimulus sequencing possible. For example, in picturepriming studies in which the prime has to be masked, the sequence of priming picture, mask, and target picture, all presented within a 500-msec time frame, requires either a third page of memory or generation time far less than that possible with the Selanar system. One of the most serious limitations of the Selanar system is the inability to access the display memory. Essentially, the creation of animated sequences is not possible. Refresh rates of $1 / 60$ th sec prohibit certain kinds of experiments, for example, subliminal priming experiments in which a prime stimulus is presented for less than $10 \mathrm{msec}$ and is then masked. The $60-\mathrm{Hz}$ raster system puts a lower limit of $16.67 \mathrm{msec}$ on stimulus presentation. To transform an image on our system, the image must be redrawn after its parameters are respecified (e.g., scaling, translation). This respecification is a time-consuming process and is one of the reasons that animation is seriously limited with this 
system. With the intensity control used to vary brightness according to lighting conditions, the decay rate of the P4 phosphor is adequate. Using PIN photodiodes connected to a 50-MHz-bandwidth oscilloscope with an image displayed at maximum brightness on a dark screen in a dark room, the phosphor decays to within $1 \%$ of darkness in 2-3 msec.

The upward bound of disk storage capacity is set by the storage medium (floppy vs. hard disk). Finally, as with any set of coordinates that are hardware-dependent, this set can be transported to any other coordinate-based graphics system and retranslated for display. This process is not always trivial. Finally, for this particular hardware system, software had to be developed, resulting in a tradeoff between the effort expended to achieve a working system and the extra purchase cost of a software/hardware package.

The Selanar system has a rather high performance/cost ratio. With a highly efficient and general-purpose (experimentation, word processing, data analysis) microcomputer system, the Selanar graphics board was the only hardware needed to add graphics to the system. Many hardware functions are included on the Selanar board, although an operating system had to be written according to the features described in the operating manual. However, most other graphics systems are weak in software support, and when software is available, there typically is extra cost involved and the software is not always available in the programming language of choice.

\section{Experimental Uses}

The Selanar system can be used for experiments that involve timed displays of alphanumeric characters, geometric figures, line drawings, and scenes. Although the Selanar system cannot produce rapid animation sequences, some other systems can if the stimuli are not too complex. As long as the display times do not need to be less than $16 \mathrm{msec}$ (as limited by a $60-\mathrm{Hz}$ refresh rate), then a sequence of stimuli may be presented with the graphics system. For example, consider a word-priming study. First, a warning signal is presented (a centered plus sign). After a 500-msec interval, the screen is blanked and the prime stimulus (word/nonword) is presented at the center of the screen. After another variable interval (200$2,000 \mathrm{msec}$ ), a second letter string is presented where the first stimulus appeared. If the first or second stimulus is to be masked, the mask could be stored on one of the pages of memory. This method is preferable to "writing" the mask anew each trial if the mask is complex. The words themselves do not need to be stored on a page of memory but can be drawn in a specific size or shape to control for visual angle of viewing or in a vector mode that would degrade the stimulus (dotted type).

Picture-priming experiments (Bonnefil, Kaye, \& Herskovits, 1984) also can be conducted. Prior to each trial, the prime and target pictures (lines drawings) are drawn to each page of memory with the video off. During the trial, each page is turned on (with 3-4 msec of drawing time rather than the $900 \mathrm{msec}$ or so that would be required to draw the picture in real time) at the appropriate time. Subjects' responses and latencies can be recorded by interfacing appropriate software routines with graphics presentation.

Experiments can be performed to study: (1) simple reaction time (RT), using brief presentations of any character or simple stimulus; (2) choice RT, with location, orientation, size, shape, shading (or color in other systems)-even selective-looking paradigms may be used with infant subjects; (3) mental rotation, with a stimulus displayed visually at any angle to be compared with a stimulus held in memory; (4) comparative judgments, with pictures compared on any semantic dimension simultaneously or successively; (5) visual search, with letters, digits, or other symbols; (6) visual illusions, in two or three dimensions; (7) Stroop tasks (with color systems); (8) perceptual processes in reading, in which features of the text characters are varied (e.g., size, spacing); (9) scene perception and memory; and (10) a variety of psychophysical comparisons (although the limits of such work may quickly be reached because of screen-resolution requirements).

These types of experiments do not exhaust the possible applications for the graphics system; only the programmer's ingenuity and the hardware limitations (e.g., raster scan rate, character resolution) prevent the complete duplication of tachistoscope studies. Experiments that cannot be conducted with the low- and medium-cost systems we have discussed might include the requirements of: (1) rapid animation (or other data transformations, such as rotation) of high-resolution images, and (2) extremely short interstimulus intervals (less than $16 \mathrm{msec}$, as in various threshold-detection experiments). Either a high-cost vector drawing graphics system or a tachistoscope would be necessary for such experiments.

\section{SUMMARY AND CONCLUSIONS}

Tachistoscopes have provided experimental psychologists with a tool useful for studying a variety of visual information processes. This device has allowed precise control over stimulus attributes and timing sequences, and with monoptic, dichoptic, or binocular viewing. Although the tachistoscope is relatively inflexible compared with computer-based graphics systems in terms of stimulus preparation and presentation, it will be needed to conduct experiments requiring rapid presentation of stimuli with extremely short interstimulus intervals until a more affordable method is developed to present complex visual stimuli at ultra-high speeds. For a variety of other experiments, however, there are relatively low-cost graphics sytems that satisfy many of the important system criteria. We have described one such system in detail and compared it with other systems ranging in affordability.

\section{REFERENCES}

Bonnefil, V. A., Kaye, D. B., \& Herskovits, E. H. (1984, April). Semantic priming by pictures and words. Paper presented at the meeting of the American Educational Research Association, New Orleans. 
Glazenborg, G., \& Schreuder, R. (1983). Software tools for creating line drawings on a CRT. Behavior Research Methods \& Instrumentation, 15, 453-455.

MontGomery, A. A., \& Soo Hoo, G. (1982). ANIMAT: A set of programs to generate, edit, and display sequences of vector-based images. Behavior Research Methods \& Instrumentation, 14, 39-40.

\section{NOTE}

1. The Selanar graphics board is available for a variety of terminals and computers. Our choice of this Z80A-based S-100 system, the purchase of which preceded our acquisition of the graphics system, was based on a number of needs for our laboratory. The type of computer system chosen is a complex issue deserving a more complete analysis than is relevant to this paper.

(Manuscript received March 8, 1984;

revision accepted for publication November 6,1984 .) 